Frontiers in Heat and Mass Transfer

\title{
AN EFFECT OF CATTANEO CHRISTOV HEAT FLUX MODEL FOR EYRING POWELL FLUID OVER AN EXPONENTIALLY STRETCHING SHEET
}

\author{
B. Ahmad* and Z. Iqbal \\ Department of Mathematics, Faculty of Science, HITEC University, Taxila, Pakistan
}

\begin{abstract}
We examine the behavior of Cattaneo-Christov heat flux model for two-dimensional incompressible flow of Eyring Powell fluid passed over an exponentially stretching sheet. Mathematical formulation is performed by assuming boundary layer approximation. Cattaneo Christov heat flux model is applied to analyze the heat transport phenomenon. Thermal relaxation time is envisaged on the layer induced due to boundary. The governing Partial Differential equations are converted into Ordinary differential equations by the appropriate use of similarity transformation. Shooting approach is used to tackle the obtained boundary layer equations. The effects of obtained similarity parameters are plotted and discussed. Computation results reveal that fluid temperature is directly related with thermal relaxation. The comparison of results, in present situation, between Fourier's law of heat conduction and Cattaneo Christov heat flux model show that the temperature and the boundary layer (thermal) thickness are smaller in Cattaneo Christov model.
\end{abstract}

Keywords: Cattaneo-Christov heat flux model, Exponentially stretching sheet, Eyring Powell fluid, Shooting method.

\section{INTRODUCTION}

In engineering and manufacturing point of view the Rheological fluids have got much importance due to its tremendous applications. Stress and deformation rate for such fluids are not related linearly. Examples involving Rheological fluids are aerodynamic extrusion of plastic sheets, the boundary layer along a liquid film and condensation process of metallic plate in a cooling bath and glass, pulps and also in polymer industries.

Due to their miscellaneous major derivation from the Newtonian fluids, Rheological fluids cannot be studied by employing a single constitutive equation. Therefore the models of Rheological fluids studied by Wang and Tan (2008), Fetecau et al. (2010), Sajid and Hayat, (2008), Hayat et al. (2012) split into three groups that is differential, integral and rate types. The Eyring-Powell fluid model analyzed by Powell and Eyring (1944) has gained much importance due to its convinced rewards over the power law model and additionally due to this model the conduct of Newtonian fluid for less and high shear rates. It describes that the shear of non-Newtonian fluid can be obtained from rate procedure hypothesis. It portraits the conduct of polymer solutions and viscoelastic suspensions on a broad range of shear rates discussed by Eldabe et al. (2003). Hayat et al. (2012) investigated the behavior of Eyring-Powell fluid for two-dimensional flow when the surface is moving continuously. Islam et al. (2009) applies Homotopy perturbation technique on Eyring-Powell model with slider bearing lubricated. Patel and Timol (2009) analyze numerically the flow of Eyring-Powell model when it passes on a wedge. Crane (1822) in his research article started the work for obtaining exact solution of viscous flow over a sheet which is stretched linearly. After this milestone achievement by crane a lot of research has been done by considering different natures of fluid with different imposed conditions like blowing/suction, heat as well as mass transfer, magnetohydrodynamics, different stretching velocities of surface.

The Fourier's (1822) heat conduction law is the main successfully implemented model for the depiction of heat transfer methods in a variety of applicable conditions. In spite of this reality it has a chief drawback that it gives a parabolic energy equation for the temperature field and for this reason it disagrees with the law of causality. Cattaneo (1948) in his well-known paper recommended a successful modification of Fourier's model by including an important aspect of thermal relaxation time. This special aspect for temperature field gives hyperbolic energy equation and it permits for the shipping of heat through the circulation of thermal waves which have finite speed. Christov (2009) replaced the time derivative in Maxwell-Cattaneo's model with the Oldroyd's upper-convected derivative in order to preserve the material-invariant formulation and this model is known as

*Corresponding author Email: bilalahmadgondal@yahoo.com 
Cattaneo-Christov heat flux model. Ciarletta and Straughan (2010) established the uniqueness of the solutions for the Cattaneo-Christov equations. Straughan (2010) studied the Structural solidity and individuality of the Cattaneo-Christov. Recently Han et al. (2014) studied the behavior of slip flow and heat transfer in Maxwell fluid by considering Cattaneo-Christov model. The governing equations are solved analytically by means of HAM. They compare their result numerically by using finite difference method.

The present paper is concerned with the analysis of Cattaneo Christov heat flux model on Eyring Powell fluid when it is passed on sheet which is stretched exponentially. The Rheological fluid flows on the sheets, which are stretched have been noticed and analyzed by many researchers. Aksoy et.al (2007) studies the stretching sheet solution of boundary layer equation of second grade fluid. Rashidi in (2010) applies differential transform method and Pade approximation method for MHD flow of thin elastic liquid films on horizontal stretching sheet. Wubshet Ibrahim (2016) studied MHD stagnation point flow of UCM fluid in the presence of nanofluid. Mahapatra et al. (2014) discussed the effect of MHD on indirect natural convection flow above a horizontal hot flat plate. In addition to stretching sheet an important feature of exponentially stretching sheets is useful in different fields like industries and engineering methods such as filament from a die, blowing of glass, continuous casting, cooling of an infinite metallic in cooling bath etc. Bidin and Nazar (2009) considered the exponential stretching surface to study the thermal radiation on laminar flow and heat transfer. Later on Sajid and Hayat (2010) derived the analytic solution of this problem. Mukhopadhyay and Gorla (2012) discussed the impact of partial slip on the over an exponentially stretching sheet. Bhattacharyya (2012) studied the mass transfer on boundary layer flow past on a sheet which is stretched exponentially. In (2013) Swati Mukhopadhyay discussed the exponentially stretching sheet which is embedded in thermal startified medium with MHD on boundary layer flow. In (2013) Jat and Gopi Chand analyzed the Viscous Dissipation and Radiation Effects of exponentially stretching sheet on MHD flow and heat transfer. Recently, Junaid et al. (2015) examined the effect of exponentially stretching sheet for viscoelastic flows by considering Cattaneo Christov heat flux model. The rare information about Eyring Powell fluid model leads us for this work. The considered model has upper hand on the other Rheological fluids because it is figured out from kinetic theory instead of the empirical relation. Additionally it shrinks the Newtonian conduct at low and high shear rate. Here we apply give the numerical technique for obtaining the solutions. Involved parameters are plotted and discussed.

\section{PROBLEM FORMULATION}

Let us suppose a time independent two dimensional incompressible flow of Eyring Powell fluid on a flexible surface placed at $y=0$. The surface is stretched exponentially with velocity $U_{w}(x)=U_{0} e^{x / L}$. A heat source $T_{w}=T_{\infty}+T_{0} e^{A x / 2 L}$ which is non uniform in nature is applied. $T_{0}$ denotes the heating/cooling reference temperature. The stress tensor in an Eyring-Powell fluid model is

$\tau_{i j}=\mu \frac{\partial u_{i}}{\partial x_{j}}+\frac{1}{\beta} \sinh ^{-1}\left(\frac{1}{C} \frac{\partial u_{i}}{\partial x_{j}}\right)$

where $\quad \mu$ is the viscosity coefficient, $\beta$ and $C$ are the characteristics of Eyring-Powell model. By using $\sinh ^{-1}\left(\frac{1}{C} \frac{\partial u_{i}}{\partial x_{j}}\right) \cong \frac{1}{C} \frac{\partial u_{i}}{\partial x_{j}}-\frac{1}{6}\left(\frac{1}{C} \frac{\partial u_{i}}{\partial x_{j}}\right)^{3}, \quad\left|\frac{1}{C} \frac{\partial u_{i}}{\partial x_{j}}\right|<<1$,

And refer to the boundary layer approximation, the governing equations for the two dimensional flow and heat exchange phenomenon of Eyring-Powell fluid by keeping density as constant are

$\frac{\partial u}{\partial x}+\frac{\partial v}{\partial y}=0$

$u \frac{\partial u}{\partial x}+v \frac{\partial u}{\partial y}=\left(v+\frac{1}{\rho \beta C}\right) \frac{\partial^{2} u}{\partial y^{2}}-\frac{1}{2 \rho \beta C^{3}}\left(\frac{\partial u}{\partial y}\right)^{2} \frac{\partial^{2} u}{\partial y^{2}}$

$\left(\rho c_{p}\right)\left(u \frac{\partial T}{\partial x}+v \frac{\partial T}{\partial y}\right)=-\nabla \cdot q$

where $u$ and $v$ represents components of velocity along $x$ - and $y$-directions respectively, $v=\mu / \rho$ denotes the kinematic viscosity. $T$ and $T_{\infty}$ represents fluid temperature and temperature at infinity. $c_{p}$ is the specific heat $q$ represents fluid temperature heat flux respectively, which obeys the given relationship.

$q+\lambda\left(\frac{\partial q}{\partial t}+\mathbf{V} \cdot \nabla \mathbf{q}-\mathbf{q} \cdot \nabla \mathbf{V}+(\nabla \cdot \mathbf{V}) \mathbf{q}\right)=-k \nabla T$

where the parameter $\lambda$ denotes the relaxation time for heat flux, $k$ and $\mathbf{V}$ represents the thermal conductivity and velocity vector respectively. Simplifying Eqs (4) and (5) to get rid of $q$ we get

$u \frac{\partial T}{\partial x}+v \frac{\partial T}{\partial y}+\lambda\left[\begin{array}{c}\left.u \frac{\partial u}{\partial x}+v \frac{\partial u}{\partial y}\right) \frac{\partial T}{\partial x}+ \\ \left.u \frac{\partial v}{\partial x}+v \frac{\partial v}{\partial y}\right) \frac{\partial T}{\partial y} \\ +u^{2} \frac{\partial^{2} T}{\partial x^{2}}+v^{2} \frac{\partial^{2} T}{\partial y^{2}}+ \\ 2 u v \frac{\partial^{2} T}{\partial x \partial y}\end{array}\right]=\alpha \frac{\partial^{2} T}{\partial y^{2}}$,

where $\alpha\left(=k / \rho c_{p}\right)$ is the thermal diffusivity and $\lambda$ is relaxation time for heat flux. The boundary conditions will be

at $y=0$

$u=U_{w}(x)=U_{0} e^{x / L}, \quad v=0, \quad T=T_{w}(x)=T_{\infty}+T_{0} e^{A x / 2 L}$

$u \rightarrow 0, \quad T \rightarrow T_{\infty}$ as $y \rightarrow \infty$

where $A$ is the temperature exponent. Using the similarity transformations

$\eta=\sqrt{\frac{U_{0}}{2 v L}} e^{x / 2 L} y, u=U_{0} e^{x / L} f^{\prime}, v=-\sqrt{\frac{v U_{0}}{2 L}} e^{x / 2 L}\left(f+\eta f^{\prime}\right)$,

$\theta=\frac{T-T_{\infty}}{T_{w}-T_{\infty}}$

equation (2) is satisfied and eqs. (3) - (7) take the following forms

$$
\begin{aligned}
& (1+\varepsilon) f^{\prime \prime \prime}+f f^{\prime \prime}-2 f^{\prime 2}-\varepsilon \delta f^{\prime \prime 2} f^{\prime \prime \prime}=0 \\
& \frac{1}{\operatorname{Pr}} \theta^{\prime \prime}+f \theta^{\prime}-A f^{\prime} \theta+\frac{\Lambda}{2}\left[\begin{array}{l}
A f f^{\prime \prime} \theta-A(2+A) f^{\prime 2} \theta \\
+(1+2 A) f f^{\prime} \theta^{\prime}-f^{2} \theta^{\prime \prime}
\end{array}\right]=0 \\
& f(0)=0, f^{\prime}(0)=1, \theta(0)=1, f^{\prime}(\infty) \rightarrow 0, \theta(\infty) \rightarrow 0,
\end{aligned}
$$

where $\varepsilon$ and $\delta$ are the characteristic parameters of Eyring Powell fluid model, $\Lambda$ is the relaxation time and $\operatorname{Pr}$ is the Prandtl number and is defined as 


$$
\varepsilon=\frac{1}{\mu \beta C}, \delta=\frac{U_{0}^{3}}{4 \nu L C^{2}} e^{\frac{3 x}{L}}, \quad \Lambda=\frac{\lambda U_{0} e^{x / L}}{L}, \quad \operatorname{Pr}=v / \alpha
$$

Expression for skin friction and coefficient $C_{f}$ and local Nusselt number $N u_{x}$ are

$$
\begin{aligned}
& \sqrt{2}\left(\operatorname{Re}_{x}\right)^{1 / 2} C_{f}=(1+\varepsilon) f^{\prime \prime}(0)-\frac{\varepsilon \delta}{3}\left(f^{\prime \prime}(0)\right)^{3} \\
& \left(\operatorname{Re}_{x}\right)^{-1 / 2} N u_{x}=-\frac{x}{\sqrt{2} L} \theta^{\prime}(0)
\end{aligned}
$$

where $\operatorname{Re}_{x}=U_{0} e^{x / L} L / v$ is the local Reynolds number.

\section{NUMERICAL SOLUTION}

In this section we present the numerical procedure to solve the coupled nonlinear ordinary differential equations (9) and (10) together with the boundary conditions (11) by incorporating shooting algorithm ( RK -4). For this reason we transform higher order nonlinear ordinary differential equation into system of first order ordinary differential equation by means of the transformation

$\left(y_{1}, y_{2}, y_{3}, y_{4}, y_{5}\right)=\left(f, f^{\prime}, f^{\prime \prime}, \theta, \theta^{\prime}\right)$

this yields the following

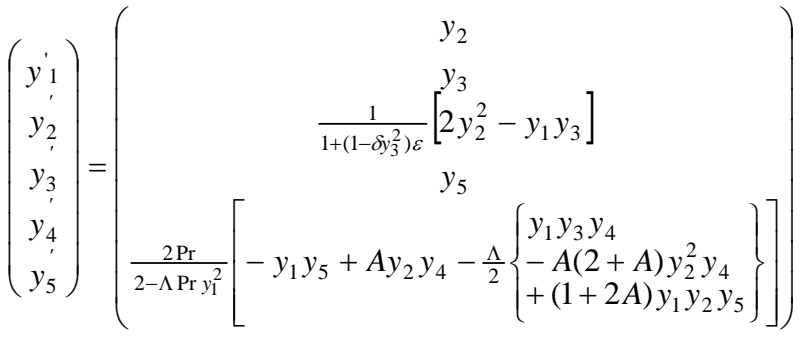

$$
\begin{aligned}
& \left(\begin{array}{l}
y_{1}(0) \\
y_{2}(0) \\
y_{3}(0) \\
y_{4}(0) \\
y_{5}(0)
\end{array}\right)=\left(\begin{array}{c}
0 \\
1 \\
f^{\prime \prime}(0) \\
1 \\
\theta^{\prime}(0)
\end{array}\right)
\end{aligned}
$$

Additionally, appropriate values of unknown initial conditions are predicted iteratively by Newton method such that solution satisfy boundary conditions at infinity with error less than $10^{-6}$.

\section{RESULTS AND DISCUSSION}

Physical behaviors of embedded parameters are discussed in this section. Fig1 depicts the effect of $\varepsilon$ (fluid material parameter) on the hydrodynamic boundary layer. It is clear from fig 1 that an increase in $\varepsilon$ results decrease in fluid viscosity. Hence the velocity of fluid increases due to decreasing viscosity. As the velocity profiles are moving away from the stretching wall when $\varepsilon$ increases which clears that the boundary layer thickness decreases and hence it is inversely related to $\boldsymbol{\epsilon}$. Fig 2 shows the effect of $\boldsymbol{\delta}$ on velocity profile. It is clear from the fig that increase in delta results an increase in viscosity and hence velocity decreases. Fig 3 shows the behavior of $\operatorname{Pr}$ (Prandtl number) on thermal boundary layer is studied in the presence and absence of $\Lambda$. It can be easily justified from fig 2 that the temperature as well as boundary layer thickness decreases in both the situations with increasing $\operatorname{Pr}$. Importantly the deviation in $\theta$ is equal in amount in
Fourier and the considered Cattaneo Christov heat flux models. Also the thermal boundary layer seems to be thinner when Pr increases. This thinner boundary layer (thermal) gives temperature profile to be steeper which point toward the bigger wall slope of temperature function. The visualization of Fig 4 indicates the action of $A$ on the temperature profile. A nice-looking fact that the temperature $\theta$ after considering negative $A$ moves first towards the highest range and then falls down exponentially to zero when $\eta$ is augmented which results reverse heat flux adjacent to the surface. When $A$ increases in positive/negative direction we observe a spiky increase in wall slope of temperature function. In Fig 5 the effect of epsilon on thermal boundary layer is viewed with and without the consideration of thermal relaxation time. Stronger viscous forces associated with larger epsilon. Fig 6 shows the effects of $\Lambda$ on the temperature distribution. We get the result that temperature $\theta$ is related inversely with $\Lambda$. Moreover temperature $\theta$ moves toward the free stream condition at shorter distance above the surface for larger $\Lambda$. Fig 7 and 9 represents the contour plot for the flow pattern for $\varepsilon=0.5$ and $\varepsilon=1.0$ respectively. Fig 8 and 10 represents the 3D plot of streams lines for $\varepsilon=0.5$ and $\varepsilon=1.0$ respectively.

Table 1 depicts the numerical values of skin friction coefficient and wall temperature gradient for different values of parameters. It is noticed from the table that skin friction and wall temperature increases as the values of characteristic parameter $\delta$ increase and there is no change in both the while increasing $\varepsilon$. Moreover there is no change in skin friction coefficient while the wall temperature coefficient decreases when thermal relaxation time $\Lambda$ is increased. The wall temperature coefficient decreases when Parndtl number and temperature exponent $A$ is incremented where as slight/no change is observed against $f^{\prime \prime}(0)$.

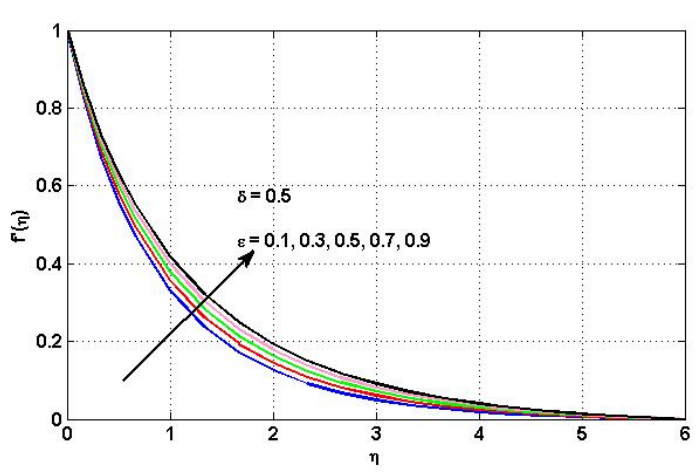

Fig. 1 Effect of $\varepsilon$ on $f^{\prime}(\eta)$.

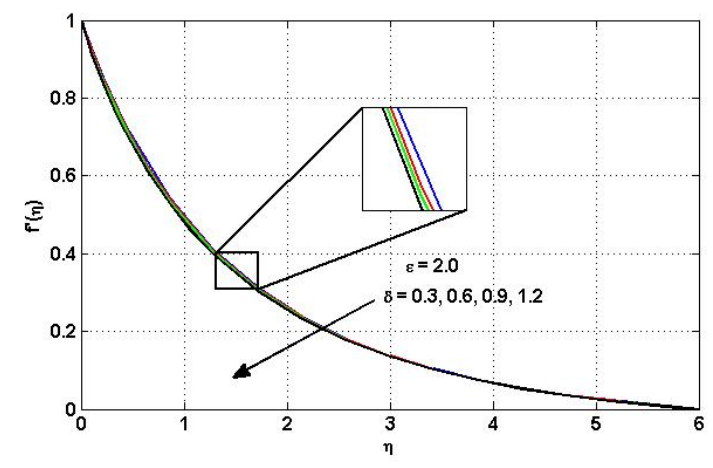

Fig. 2 Effect of $\delta$ on $f^{\prime}(\eta)$. 


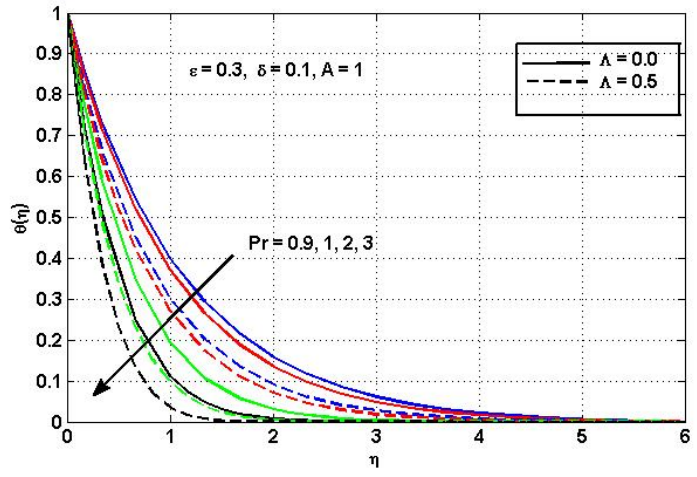

Fig. 3 Effect of $\operatorname{Pr}$ on $\theta(\eta)$

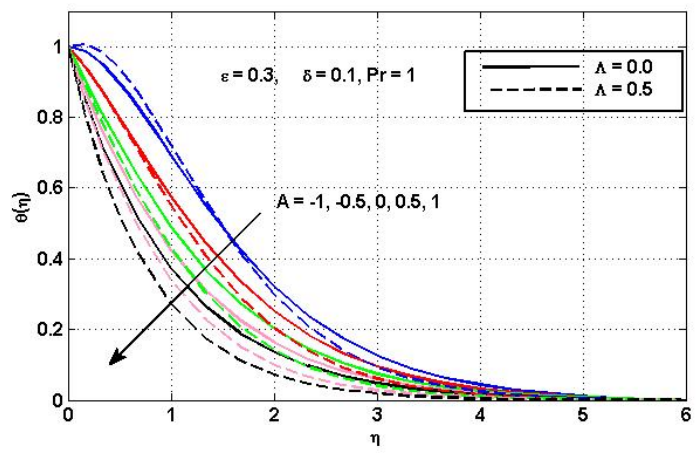

Fig. 4 Effect of $A$ on $\theta(\eta)$

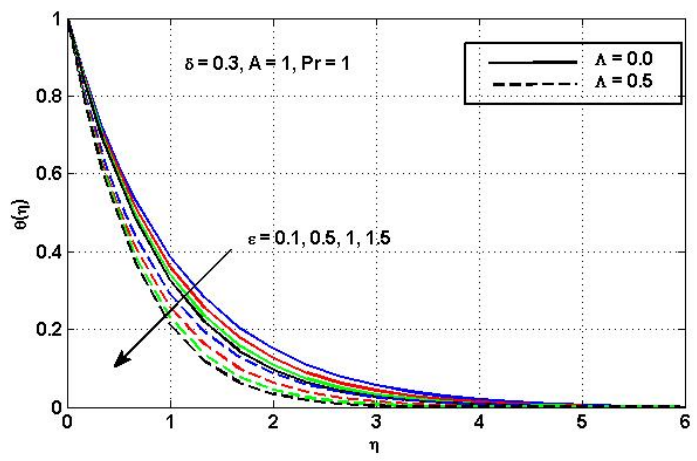

Fig. 5 Effect of $\varepsilon$ on $\theta(\eta)$

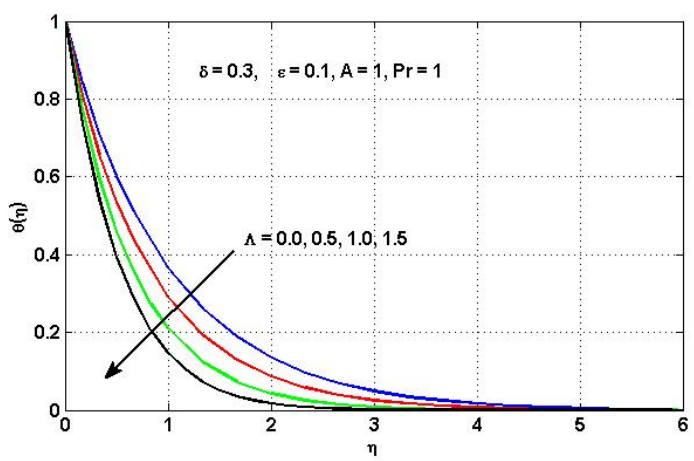

Fig. 6 Effect of $\Lambda$ on $\theta(\eta)$

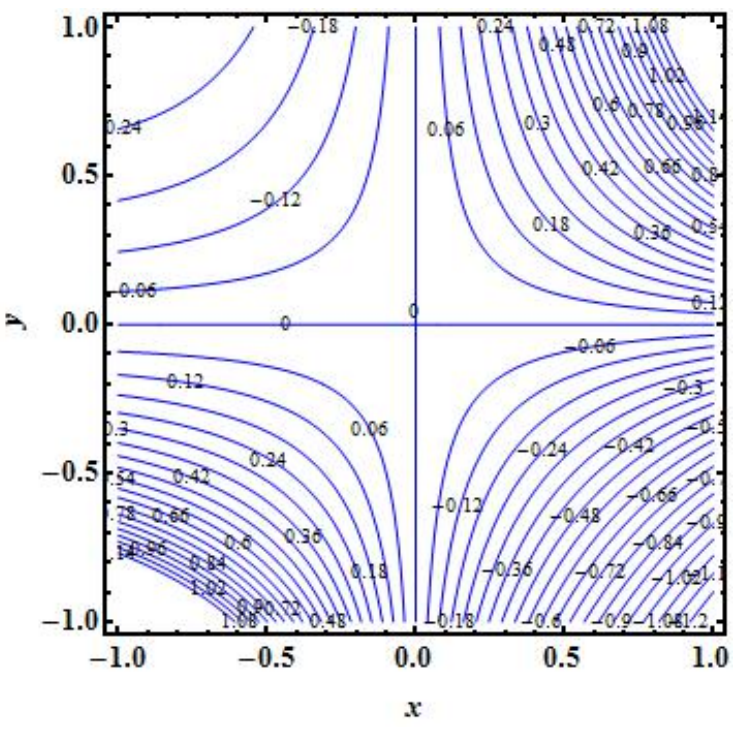

Fig. 7 Contour plot of Stream lines for $\varepsilon=0.5$

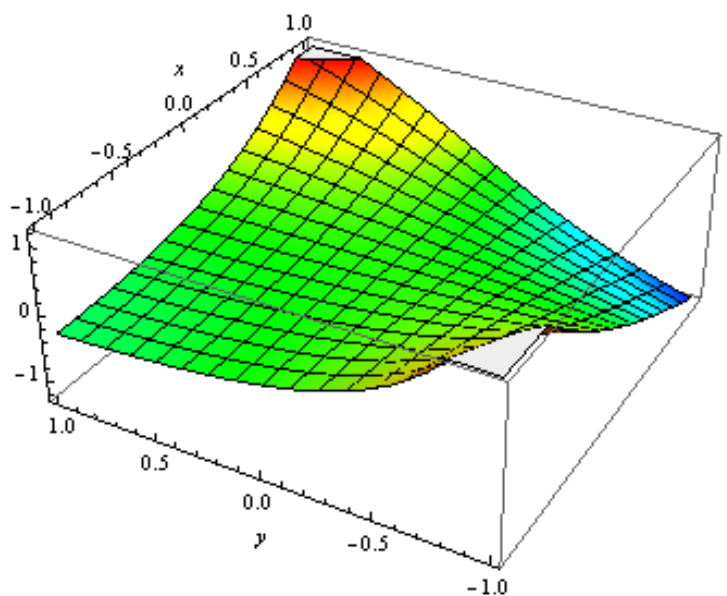

Fig. $83 \mathrm{D}$ plot of Stream lines for $\varepsilon=0.5$

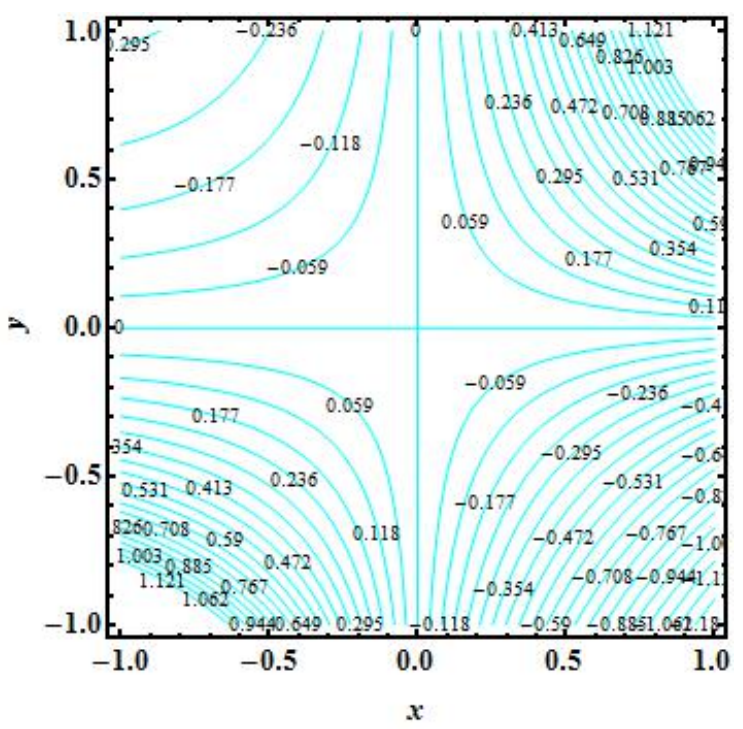

Fig. 9 Contour plot of Stream lines for $\varepsilon=1.0$ 


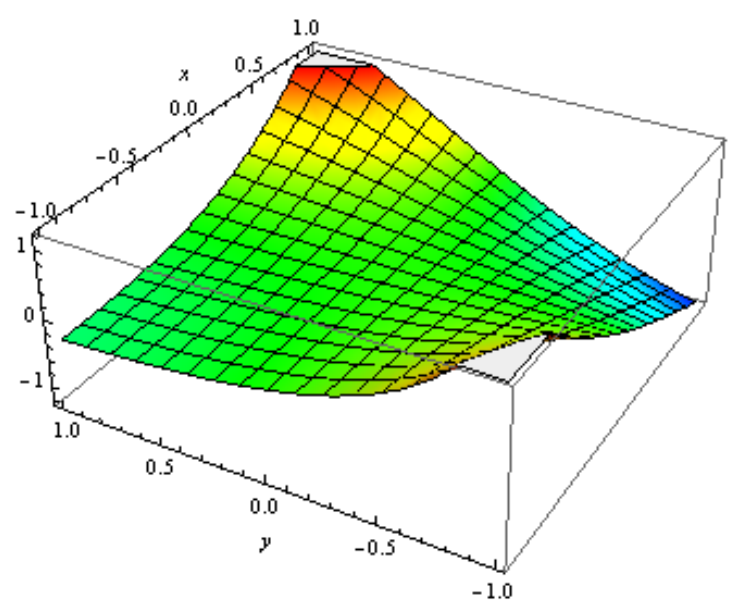

Fig. $103 \mathrm{D}$ plot of Stream lines for $\varepsilon=1.0$

Table 1 Depicts results of skin friction $-f^{\prime \prime}(0)$ and wall temperature gradient $-\theta^{\prime}(0)$ for different values of parameters.

\begin{tabular}{|l|l|l|l|r|r|l|}
\hline$\varepsilon$ & $\delta$ & $\operatorname{Pr}$ & $\Lambda$ & $A$ & $-f^{\prime \prime}(0)$ & $-\theta^{\prime}(0)$ \\
\hline 0.5 & 0.2 & 1.0 & 0.5 & -1.5 & 1.75560 & -0.750218 \\
\hline & 0.4 & & & & 1.74098 & -0.745766 \\
\hline & 0.6 & & & & 1.72514 & -0.74094 \\
\hline & 0.8 & & & & 1.70757 & -0.735613 \\
\hline 0.5 & 0.5 & 1.0 & 0.2 & -1.5 & 1.73324 & -0.563859 \\
\hline & & & 0.4 & & 1.73324 & -0.682238 \\
\hline & & & 0.6 & & 1.73324 & -0.805835 \\
\hline & & & 0.8 & & 1.73324 & -0.934266 \\
\hline 0.5 & 0.5 & 0.5 & 0.5 & -1.5 & 1.73324 & -0.363801 \\
\hline & & 1.0 & & & 1.73323 & -0.743406 \\
\hline & & 1.5 & & & 1.73323 & -1.02065 \\
\hline & & 2.0 & & & 1.73323 & -1.25819 \\
\hline 0.2 & 0.5 & 1.0 & 0.5 & -1.5 & 1.73323 & -0.743406 \\
\hline 0.4 & & & & & 1.73323 & -0.743406 \\
\hline 0.6 & & & & & 1.73323 & -0.743406 \\
\hline 0.8 & & & & & 1.73323 & -0.743406 \\
\hline 0.5 & 0.5 & 1.0 & 0.5 & -1.5 & 1.73323 & 0.743406 \\
\hline & & & & -0.5 & 1.73324 & -0.324314 \\
\hline & & & & 0.5 & 1.73324 & -1.04976 \\
\hline & & & & 1.5 & 1.73324 & -1.36642 \\
\hline
\end{tabular}

\section{CONCLUDING REMARKS}

We have investigated the characteristics of boundary layer flow of Eyring Powell fluid on the surface which is stretching exponentially. Cattaneo Christov model for heat flux is imposed to disclose the heat transfer characteristics of variable thermal conductivity of viscoelastic fluid. The main observations are summarized as follows:

- Hydrodynamic boundary layer shows opposite behavior for both fluid parameters $\varepsilon$ and $\delta$.

- $\quad$ Temperature and thermal boundary layer thickness are increasing functions of relaxation time $\Lambda$.

- For negative temperature exponent an attractive phenomenon named as Sparrow Gregg Hills is observed for temperature division.

- Parameters behavior shows that temperature distribution is lower in Cattaneo Christov heat flux model as compared to Fourier law of heat conduction.

\section{NOMENCLATURE}

$\begin{array}{ll}v & \text { Kinematic viscosity }(\mu / \rho) \\ \alpha & \text { Thermal diffusivity }\left(k / \rho c_{p}\right) \\ \varepsilon & \text { Characteristic parameters of Eyring Powell fluid }\left(\frac{1}{\mu \beta C}\right) \\ \delta & \text { Characteristic parameters Eyring Powell fluid }\left(\frac{U_{0}^{3}}{4 v L C^{2}} e^{\frac{3 x}{L}}\right) \\ \Lambda & \text { Relaxation time }\left(\frac{\lambda U_{0} e^{x / L}}{L}\right) \\ \operatorname{Pr} & \text { Prandtl number }(v / \alpha) \\ \operatorname{Re}_{x} & \text { Local Reynolds number }\left(U_{0} e^{x / L} L / v\right)\end{array}$

\section{REFERENCES}

Ahmad Khan, J., Mustafa, M., Hayat, T., Alsaedi, A., 2015, "Numerical Study of Cattaneo Christov Heat Flux Model for Viscoelastic Flow Due to an Exponentially Stretching Surface,” PLoS ONE, 10(9). http://dx.doi.org/10.1371/journal.pone.0137363

Aksoy, Y., Pakdemirli, M., Khalique, C. M., 2007, "Boundary Layer Equations and Stretching Sheet Solutions for the Modified Second Grade Fluid,” International Journal of Engineering Science, 45(10), 829-841 http://dx.doi.org/10.1016/j.ijengsci.2007.05.006

Bidin, B., Nazar, R., 2009, Numerical Solution of the Boundary Layer Flow Over an Exponentially Stretching Sheet with Thermal Radiation,” Eur. J. Sci. Res, 33, 710-717.

Bhattacharyya, K., 2012, "Steady Boundary Layer Flow and Reactive Mass Transfer Past an Exponentially Stretching Surface in an Exponentially Moving Free Stream,” J. Egypt. Math. Soc., 20, 223-228. http://dx.doi.org/10.1016/j.joems.2012.08.018

Cattaneo, C., 1948, "Sulla conduzionedelcalore, AttiSemin,” Mat. Fis. Univ. Modena Reggio Emilia, 3, 83-101.

Christov, C.I., 2008, “On Frame in Different Formulation of The Maxwell-Cattaneo Model of Finite-speed Heat Conduction,” Mechanics Research Communications, 36, 481-486.

http://dx.doi.org/10.1016/j.mechrescom.2008.11.003

Ciarletta, M., Straughan, B., 2010, "Uniqueness and Structural Stability for the Cattaneo-Christov Equations,” Mechanics Research Communications, 37, 445-447. http://dx.doi.org/10.1016/j.mechrescom.2010.06.002

Crane, L.J., 1822, “Flow Past a Stretching Plate,” J. Appl Math and Phy, 21, 645-647.

Eldabe, N.T.M., Hassan, A.A., Mohamed, B., Mona, A.A., 2003, "Effect of Couple Stresses on the MHD of a Non-Newtonian Unsteady Flow Between Two Parallel Porous Plates,” Zeitschrift Naturforschung A, 58, 204-210. 
Fetecau C., Zierep, J., Bohning, R., 2010, “On the Energetic Balance for the Flow of an Oldroyd-B Fluid Due to a Flat Plate Subject to a Time-Dependent Shear Stress,” Comput Math Appl, 60, 74-82. http://dx.doi.org/10.1016/j.camwa.2010.04.031

Fourier, J.B.J., 1822, “Théorie Analytique De La Chaleur (Paris) F. Diot”.

Han, S., Zheng, L., Li, C., Zhang, X., 2014, “Coupled Flow and Heat Transfer in Viscoelastic Fluid with Cattaneo-Christov Heat Flux Model,” Appl. Math Letters, 38, 87-93.

http://dx.doi.org/10.1016/j.aml.2014.07.013

Hayat, T., Iqbal, Z., Qasim, M., Obaidat, S., 2012, "Steady Flow of an Eyring Powell Fluid Over a Moving Surface with Convective Boundary Conditions,” Intl. J. Heat and Mass Transfer, 55, 1817-1822. http://dx.doi.org/10.1016/j.ijheatmasstransfer.2011.10.046

Hayat, T., Iqbal, Z., Mustafa, M., 2012, "Flow of a Second Grade Fluid Over a Stretching Surface with Newtonian Heating”, Journal of Mechanics 28, 209-216.

http://dx.doi.org/10.1017/jmech.2012.21

Islam, S., Shah, A., Zhou, C.Y., Ali, I., 2009, "Homotopy Perturbation Analysis of Slider Bearing with Powell-Eyring Fluid,” Zeitschrift für angewandte Mathematik und Physikm, 60, 1178-1193.

http://dx.doi.org/10.1007/s00033-009-7034-9

Jat, R.N., Gopi Chand, 2013, "MHD Flow and Heat Transfer over an Exponentially Stretching Sheet with Viscous Dissipation and Radiation Effects,” Applied Mathematical Sciences, 7(4), 167-180.

Mahapatra, T.R., Sidui,S., Nandy, S.K., 2014, "Effect of Magnetic Field on Indirect Natural Convection Flow above a Horizontal Hot Flat Plate,” Frontiers in Heat and Mass Transfer, 5, 15

http://dx.doi.org/10.5098/hmt.5.15

Mukhopadhyay, M., Gorla, R.S.R., 2012, "Effects of Partial Slip on Boundary Layer Flow Past a Permeable Exponential Stretching Sheet in Presence of Thermal Radiation,” Heat Mass Transfer, 48(10), 17731781.

http://dx.doi.org/10.1007/s00231-012-1024-8
Patel, M., Timol, M.G., 2009, “Numerical Treatment of Powell-Eyring Fluid Flow Using Method of Satisfaction of Asymptotic Boundary Conditions (MSABC),” Applied Numerische Mathematik, 59, 25842592.

http://dx.doi.org/10.1016/j.apnum.2009.04.010

Powell RE, Eyring H (1944) Nature, London p. 427.

Rashidi, M.M., Keimanesh, M., 2010, “Using Differential Transform Method and Pade'Approximant for Solving MHD Flow in a Laminar Liquid Film from a Horizontal Stretching Surface,” Mathematical Problems in Engineering, 20, 491319. http://dx.doi.org/10.1155/2010/491319

Sajid, M., Iqbal, Z., Hayat, T., Obaidat, S., 2011, "Series Solution for Rotating Flow of an UCM Fluid Over a Stretching Sheet," Communications in Theoretical Physics, 56, 740-744. http://iopscience.iop.org/0253-6102/56/4/24

Sajid, M., Hayat, T., 2008, "Influence of Thermal Radiation on the Boundary Layer Flow due to an Exponentially Stretching Sheet," Int. Commun. Heat Mass Transfer, 35, 347-356.

http://dx.doi.org/10.1016/j.icheatmasstransfer.2007.08.006

Straughan, B., 2010, “Thermal Convection with the Cattaneo-Christov Model,” Intl. J. Heat and Mass Transfer, 53, 95-98. http://dx.doi.org/10.1016/j.ijheatmasstransfer.2009.10.001

Mukhopadhyay, S., 2013, "MHD Boundary Layer Flow and Heat Transfer over an Exponentially Stretching Sheet Embedded in a Thermally Stratified Medium,” Alexandria Engineering Journal, 52(3), 259-265.

http://dx.doi.org/10.1016/j.aej.2013.02.003

Wang, S., Tan, W.C., 2008, "Stability Analysis of Double-Diffusive Convection of Maxwell Fluid in a Porous Medium Heated from Below,” Phy. Letters A, 372, 3046-3050.

http://dx.doi.org/10.1016/j.physleta.2008.01.024

Ibrahim, W., 2016, "Magnetohydrodynamic(MHD) Stagnation Point Flow and Heat Transfer of Upper-Convected Maxwell Fluid Past a Stretching Sheet in the Presence of Nanoparticles with Convective Heating," Frontiers in Heat and Mass Transfer, 7, 4. http://dx.doi.org/10.5098/hmt.7.4 